\title{
THE ABSENCE OF CALCIUM BLOCKS IMPULSE-EVOKED RELEASE OF ACETYLCHOLINE BUT NOT DE NOVO FORMATION OF FUNCTIONAL NEUROMUSCULAR SYNAPTIC CONTACTS IN CULTURE ${ }^{1}$
}

\author{
LESLIE P. HENDERSON, ${ }^{2}$ MARTIN A. SMITH, AND NICHOLAS C. SPITZER \\ Department of Biology, University of California, San Diego, La Jolla, California 92093
}

Received March 21, 1984; Revised May 25, 1984; Accepted May 30, 1984

\begin{abstract}
We have examined the role of calcium-dependent, impulse-evoked release of acetylcholine (ACh) in the formation of functional neuromuscular contacts. Dissociated cell cultures prepared from Xenopus laevis embryos were maintained either in standard medium containing calcium or in calcium-free medium. Both the number of neuron-myocyte contacts and the number of terminations of neurites on myocytes were reduced in $\mathrm{Ca}$-free medium. Intracellular recordings from neuron-myocyte pairs during perfusion with a standard saline revealed that functional synaptic contacts were formed in Ca-free medium, although with a reduced frequency $(\sim 30 \%$ of controls). Postsynaptic potentials elicited by neuronal action potentials were smaller than those observed after growth in standard medium. The frequency of small, spontaneously occurring potentials was reduced by a factor of 10. Ca-free saline prevented the impulse-evoked release of transmitter from neurons in Ca-free and control cultures. Labeling with $\alpha$-bungarotoxin and iontophoretic application of $\mathrm{ACh}$ revealed no $\mathrm{ACh}$ receptor clusters in the membrane of myocytes grown in Ca-free medium. Our results suggest that evoked, vesicular release is not required for the initial formation of neuromuscular contacts, although it may be involved in further maturation of synapses. Roles for spontaneous quantal or non-quantal release have not been excluded.
\end{abstract}

The cues that govern formation of neuromuscular synapses during development are not well understood. One possibility is that particular components involved in transmission at the mature neuromuscular junction, such as impulse-evoked vesicular release or activation of postsynaptic receptors, must be functional at the time that synaptogenesis occurs for the muscle to be recognized as an appropriate target. It appears that rapid ion fluxes mediated by postsynaptic acetylcholine receptors (AChRs) are not required since the presence of reversible neuromuscular blocking agents does not block nerve-induced aggregation of AChR nor the establishment of functional neuromuscular connections (Cohen, 1972; Steinbach et al., 1973; Jansen and Van Essen, 1975; Anderson et al., 1977; Kidokoro, 1980; Rubin et al., 1980). Therefore, it seems unlikely that either receptor occupation or acetylcholine (ACh)-induced electrical activity in the muscle is essential for synapse formation.

Transmitter release from growth cones has been demonstrated for neurons in culture (Hume et al., 1983; Young and Poo, 1983). The release of neurotransmitter, the concomitant release of other substances (Douglas and Poisner, 1966; Musick

\footnotetext{
${ }^{1}$ We thank Darwin Berg, John Bixby, Diane O'Dowd, and Joe Henry Steinbach for their review of this manuscript, and Rosario de Baca for her fine technical assistance and photography. This work was supported by National Institutes of Health Grant NS 15918. L. P. H. was also supported by postdoctoral grants from Bank of America-Giannini Foundation and Muscular Dystrophy Association; M. A. S. was supported by National Institutes of Health NS 12601.

${ }^{2}$ To whom correspondence should be addressed.
}

and Hubbard, 1972), or fusion of vesicle membrane with the plasmalemma (Buckley et al., 1983) might mediate target recognition and synaptogenesis. We have studied the formation of synapses between embryonic nerve and muscle cells of Xenopus laevis differentiating in culture in the absence of calcium.

Spinal cord neurons of Xenopus undergo a stereotyped sequence of developmental changes in membrane properties during differentiation in vivo. In particular, studies of the acquisition of electrical properties have shown that there are changes in the ionic dependence of the action potential recorded from neurons at different developmental stages. When cells first become capable of generating action potentials, regenerative currents depend primarily on an influx of calcium, and the impulses are of long duration. Later, action potentials become sodium dependent and are brief (Baccaglini and Spitzer, 1977). The same sequence of development also occurs in Xenopus spinal cord neurons growing in dissociated cell culture (Spitzer and Lamborghini, 1976; Willard, 1980; Blair, 1983; O’Dowd, 1983). Similar changes in the ionic dependence of the action potential have also been reported in embryonic murine neurons (Matsuda et al., 1976; Miyake, 1978) and chick neurons (MoriOkamoto et al., 1983) as well as in regenerating neurites of guinca pig dorsal root ganglion cells in vitro (Fukuda and Kameyama, 1979) and cockroach giant axons following transection (Meiri et al., 1981). These findings have led to speculation that the influx of calcium ions or the electrical event associated with the long-duration action potential may play a role in regulating normal development and regeneration (Spitzer, 1983). In contrast to changes in membrane properties 
responsible for the action potential, the ionic dependence of neuronal responses to neurotransmitters is constant from the time of onset of sensitivity (Goodman and Spitzer, 1979; Bixby and Spitzer, 1982). The development of neurotransmitter sensitivities of Xenopus spinal cord neurons in culture parallels that of neurons in vivo (Bixby and Spitzer, 1984a).

Xenopus spinal cord neurons in culture also differentiate when calcium is replaced by magnesium in the culture medium. These cells extend neurites and can generate action potentials when bathed in physiological saline. The developmental changes in the ionic dependence of the impulse are unaffected by this treatment. Moreover, the onset of neurotransmitter sensitivity is the same as in neurons grown in the presence of calcium, although its later development is retarded. The early differentiation of these membrane properties is thus largely independent of voltage-dependent calcium influx and of electrical activity (Bixby and Spitzer, 1984b).

The development of neuromuscular junctions between motor neurons and trunk myotomal muscles in Xenopus embryos has been described in vivo and is mimicked by the development of neuron-myocyte contacts in culture. Embryonic Xenopus muscle fibers, like those of fetal and newborn rat, are initially sensitive to $\mathrm{ACh}$ over their entire surface (Diamond and Miledi, 1962; Chow and Cohen, 1983). Xenopus myotomal muscle cells maintained in culture exhibit a similar pattern of sensitivity to iontophoretically applied ACh prior to innervation (Gruener and Kidokoro, 1982). This sensitivity reflects a fairly uniform distribution of $\mathrm{AChR}$, although regions of higher receptor density (hot spots) are also present (Anderson and Cohen, 1977; Anderson et al., 1977; Kidokoro and Gruener, 1982). Initial synaptic transmission occurs within minutes after the arrival of neurites, both in vivo and in culture (Blackshaw and Warner, 1976; Kullberg et al., 1977; Kidokoro and Yeh, 1982). Innervation induces reorganization of receptors into regions of high density at sites of contact by neurites (Anderson and Cohen, 1977; Kidokoro et al., 1980; Chow and Cohen, 1983). Synaptic transmission, however, precedes this reorganization both in Xenopus (Blackshaw and Warner, 1976; Kullberg et al., 1977; Anderson et al., 1979; Kidokoro et al., 1980; Chow and Cohen, 1983) and in rat (Bennett and Pettigrew, 1974; Bevan and Steinbach, 1977). The increase in the density of receptors in regions of nerve-muscle contact is reflected in an increase in the amplitude of miniature endplate potentials (MEPPs) and junctional sensitivity to $\mathrm{ACh}$ and a decrease in the time to peak of postsynaptic responses. After the onset of synaptic transmission, presynaptic changes also occur. There is an increase in the frequency of MEPPs (Anderson et al., 1979; Kidokoro et al., 1980) and concomitant development of presynaptic morphological specializations (Weldon and Cohen, 1979; Nakajima et al., 1980).

Impulse-evoked vesicular release of $\mathrm{ACh}$ at the adult frog neuromuscular junction is blocked by high concentrations of magnesium which antagonize calcium entry (del Castillo and Katz, 1954). Accordingly, we have asked whether synapse formation between spinal cord neurons and myocytes is affected by the absence of calcium and blockade of evoked release. Our results indicate that functional synaptic contacts are formed between nerve and muscle cells in calcium-free medium, but at a lower frequency and with a lower efficacy than in standard medium.

\section{Materials and Methods}

Cultures. Xenopus laevis embryos were obtained by conventional breeding procedures and staged according to the table of Nieuwkoop and Faber (1956). Dissociated cell cultures were prepared by methods modified from Spitzer and Lamborghini (1976) and Blair (1983). The neural plate and surrounding mesoderm were dissected from stage 15 embryos and dissociated for 10 to $15 \mathrm{~min}$ in $\mathrm{Ca}$ - and $\mathrm{Mg}$-free Steinberg's solution containing $0.4 \mathrm{mM}$ EDTA. Single cell suspensions were transferred via a fine glass capillary to $60-\mathrm{mm}$ tissue culture dishes containing approximately $3 \mathrm{ml}$ of either a modified Steinberg's solution, consisting of $58.2 \mathrm{~mm} \mathrm{NaCl}, 0.7 \mathrm{mM} \mathrm{KCl}, 1.3 \mathrm{~mm} \mathrm{MgSO}_{4}, 10.0 \mathrm{~mm}$ $\mathrm{CaCl}_{2}, 4.6 \mathrm{mM}$ Tris base, $\mathrm{pH} 7.8$ (standard medium), or an identical solution, in which $10 \mathrm{mM} \mathrm{MgCl}$ was substituted for $\mathrm{CaCl}_{2}$ and to which $1 \mathrm{mM}$ EGTA was added (Ca-free medium; see Bixby and Spitzer, 1984b). One embryo per dish was plated and cells were maintained in the same medium until the time of an experiment.

Morphological measurements. Cultures were examined under phase contrast optics with a Zeiss inverted microscope at $\times 160$. Distances between myocytes and neurons were measured using an eyepiece reticule marked with concentric rings.

Physiological recordings. Microelectrodes were prepared from standard ( $1.0 \mathrm{~mm}$ outer diameter $/ 0.58 \mathrm{~mm}$ inner diameter) glass capillary (100 to $300 \mathrm{megohms})$ or from thin-walled $(1.0 \mathrm{~mm}$ outer diameter $0.75 \mathrm{~mm}$ inner diameter) capillary tubing ( 40 to 80 megohms) and filled with $1 \mathrm{M}$ potassium acetate. Cells were viewed under Zeiss phase contrast optics with a $\times 40$ water immersion objective. Unless otherwise stated, all electrophysiological recordings were made in standard saline perfused continuously throughout the experiment. Recordings from cultures grown in Ca-free medium were made from 0 to $2 \mathrm{hr}$ after changing to saline (standard saline: $125 \mathrm{~mm} \mathrm{NaCl}, 10 \mathrm{mM} \mathrm{CaCl}_{2}, 3 \mathrm{mM}$ $\mathrm{KCl}, 5$ mm HEPES, $\mathrm{pH}$ 7.4; magnesium saline: 10 mm $\mathrm{MgCl}_{2}$ substituted for $10 \mathrm{mM} \mathrm{CaCl}_{2}$ ). Techniques for voltage, current, and conductance measurements have been described previously (Bixby and Spitzer, 1982). Cells were impaled between 15 and $27 \mathrm{hr}$ in culture, corresponding to developmental stages 28 to 34 (Nieuwkoop and Faber, 1956).

Myocytes grown either in standard or in Ca-free medium had resting potentials as large as $-80 \mathrm{mV}$, although many of the values recorded were smaller. Myocytes with low resting potentials exhibited spontaneous and evoked responses when held at $-80 \mathrm{mV}$; both neurons and myocytes were routinely held at this potential. In the absence of postsynaptic activity, pairs of cells had to meet the following criteria to be accepted for data analysis: (1) stimulation of the "presynaptic" neuron produced an action potential in that cell, (2) the myocyte was depolarized by bath application of ACh (100 $\mu \mathrm{M}$ or less), and (3) neurites were observed to remain intact during the course of recording.

Application of transmitters. ACh $(0.1$ to $100 \mu \mathrm{M}), \gamma$-aminobutyric acid (GABA; $100 \mu \mathrm{M})$, glutamate $(100 \mu \mathrm{M})$, and $d$-tubocurarine $(50 \mu \mathrm{M})$ were prepared in standard or in magnesium saline and bath applied.

The neurotransmitter sensitivity of neurite-contacted and noncontacted regions of myocyte membranes was also measured by iontophoretic application of $\mathrm{ACh}$. Myocytes were impaled with a recording electrode and approached with an iontophoretic pipette $(3 \mathrm{M} \mathrm{AChCl}$; 1000 to 1800 megohms). A steady braking current of 0 to $0.3 \mathrm{nA}$ was maintained, and pulses of $10 \mathrm{msec}$ duration and varying amplitude were applied until a response of $5 \mathrm{mV}$ was elicited. This procedure was repeated at various sites on the myocyte membrane. Sensitivity to $\mathrm{ACh}$ was expressed as millivolts per picocoulomb for each site tested. Myocytes were held at $-80 \mathrm{mV}$ throughout the measurement of sensitivity. All measurements from a single myocyte were obtained with the same iontophoretic pipette and braking current. Variations in the ability of different pipettes to pass current and in the amount of braking current applied could produce differences from cell to cell that do not accurately reflect differences in sensitivity. Therefore, the sensitivities of regions of noncontacted membrane were compared to those contacted by neurites for each myocyte and expressed as a ratio. These ratios were then pooled for comparison of cells grown in the presence or absence of calcium.

Labeling with $\alpha$-bungarotoxin. $\alpha$-Bungarotoxin $(\alpha$-BGT) was purified from the venom of Bungarus multicinctus as previously described (Ravdin and Berg, 1979). Rhodamine-labeled $\alpha$-BGT (R- $\alpha$-BGT) was prepared after the method of Ravdin and Axelrod (1977). [ $\left.{ }^{125} \mathrm{I}\right] \mathrm{di}-$ iodo$\alpha$-BGT was prepared and isolated with initial specific activities of 250 to $300 \mathrm{Ci} / \mathrm{mmol}$ according to the method of Vogel et al. (1972).

For fluorescent labeling experiments, cultures were incubated according to growth conditions in either standard or Ca-free medium containing $50 \mathrm{nM} \mathrm{R}-\alpha$-BGT and $2 \mathrm{mg} / \mathrm{ml}$ of bovine serum albumin for $1 \mathrm{hr}$ at $20^{\circ} \mathrm{C}$ followed by washing in several changes of the appropriate medium over a period of $30 \mathrm{~min}$. Cells under coverslips were examined by fluorescence microscopy. Labeling of AChR on Xenopus myocytes by R- $\alpha$-BGT was specific in that it could be blocked by inclusion of either $5 \times 10^{-6} \mathrm{M}$ unlabeled $\alpha$-BG' $\mathrm{B}$ or $1 \times 10^{-4} \mathrm{M} d$-tubocurarine in the labeling solution. 

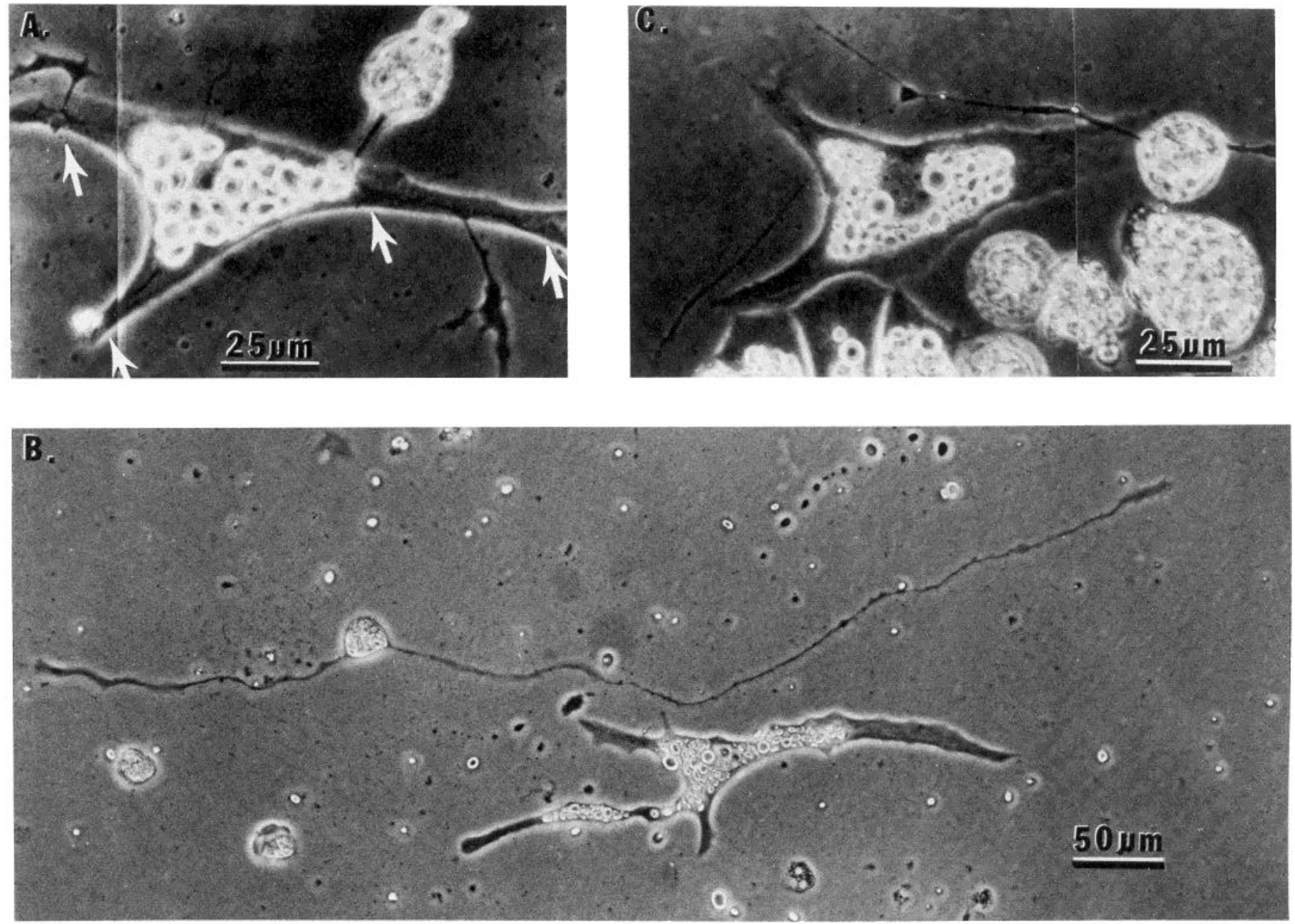

Figure 1. Patterns of neurite growth and myocyte contact in standard cultures $(A)$ and Ca-free cultures $(B$ and $C)$. $A$, Cells were plated in Steinberg's solution containing $10 \mathrm{mM} \mathrm{CaCl}_{2}$. Neurons in the vicinity of myocytes frequently extended neurites which grew toward myocytes and terminated on them (arrows). $B$ and $C$, Cells were plated in Steinberg's solution containing $0 \mathrm{mM} \mathrm{CaCl}_{2} / 10 \mathrm{mM} \mathrm{MgCl}_{2} / 1 \mathrm{~mm} \mathrm{EGTA}$. B, Neurons extended neurites that were substantially longer but contacted myocytes less frequently than did neurites in standard cultures. $C$, Those neurites that did contact myocytes most often did not make terminations on them but continued onward and ended on unoccupied areas of the dish. Cells were approximately $24 \mathrm{hr}$ in culture.

\section{TABLE I}

Frequencies of morphological neuron-myocyte contacts and myocytespecific neurite terminations in culture

Cultures were examined at $\times 160$ under phase contrast optics. Myocytes were chosen which had neighboring neurons whose neurites grew within $60 \mu \mathrm{m}$ of the myocytes. The percentage of these cells that were contacted by neurites was tabulated. The percentages of this group of cells, i.e., contacted myocytes, on which neurites terminated was then calculated. (The normal approximation to a binomial distribution indicates values are significantly different, $p<0.001$.) For Cafree cultures, cells were plated in Steinberg's solution that contained $10 \mathrm{mM} \mathrm{MgCl}, 0 \mathrm{mM} \mathrm{CaCl}_{2}$ and $1 \mathrm{~mm}$ EGTA. For standard cultures, cells were plated in Steinberg's solution that contained $10 \mathrm{mM} \mathrm{CaCl}_{2}$.

\begin{tabular}{ccccc}
\hline \multicolumn{2}{c}{ Standard Medium } & & \multicolumn{2}{c}{ Ca-free Medium } \\
\cline { 1 - 2 } \cline { 5 - 6 } $\begin{array}{c}\text { Percentage } \\
\text { contacted }\end{array}$ & $\begin{array}{c}\text { Percentage of } \\
\text { terminations }\end{array}$ & & $\begin{array}{c}\text { Percentage } \\
\text { contacted }\end{array}$ & $\begin{array}{c}\text { Percentage of } \\
\text { terminations }\end{array}$ \\
\hline $82(90 / 110)$ & $89(80 / 90)$ & & $57(68 / 119)$ & $29(20 / 68)$ \\
\hline
\end{tabular}

The distribution of AChR on Xenopus myocytes was also assessed by light microscopic autoradiography. Cultures were incubated for $1 \mathrm{hr}$ at $20^{\circ} \mathrm{C}$ in culture medium containing $40 \mathrm{nM}\left[{ }^{125} \mathrm{I}\right] \mathrm{di}$-iodo- $\alpha$-BGT and $2 \mathrm{mg} / \mathrm{ml}$ of bovine serum albumin, conditions previously reported to saturate AChR on cultured Xenopus myocytes (Kidokoro and Gruener, 1982). Cells were washed four times over a period of $30 \mathrm{~min}$ with fresh culture medium followed by fixation for $1 \mathrm{hr}$ at $20^{\circ} \mathrm{C}$ in $2 \%$ glutaraldehyde dissolved in standard culture medium. Cells were washed several times in distilled water, dehydrated by rinsing with graded ethanol solutions, and air dried. Cultures were coated with Kodak NTB2 emulsion diluted 1:1 with distilled water, exposed for 1 to 5 days at $4^{\circ} \mathrm{C}$, and developed with Kodak D-19 developer for $2.5 \mathrm{~min}$ at $18^{\circ} \mathrm{C}$.

Materials. Glass capillary was purchased from Hilgenberg and from Frederick Haer and Co. ACh, $d$-tubocurarine, GABA, glutamate, and bovine serum albumin were purchased from Sigma Chemical Co. Bungarus multicinctus venom was obtained from the Miami Serpentarium, tetramethylrhodamine isothiocyanate was from Research Organics, Inc., and $\mathrm{Na}^{125} \mathrm{I}$ was from Amersham Corp.

\section{Results}

Patterns of growth. Spinal cord neurons differentiating in $\mathrm{Ca}$-free medium are more numerous and extend significantly longer neurites than cells differentiating in standard medium, although the number of neurites per neuron is not significantly different (Bixby and Spitzer, 1984b). Despite this, it was observed that neurons grown in $\mathrm{Ca}$-free medium did not contact myocytes as frequently as did those in standard medium. Furthermore, neuron-myocyte contacts in standard cultures had 


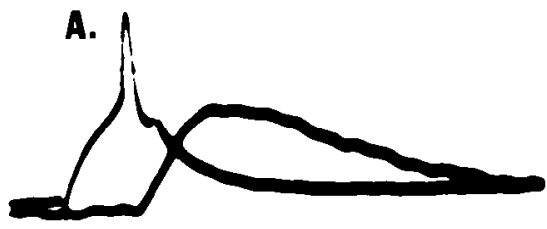

Standard Medium

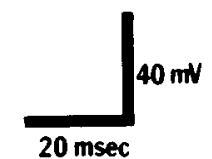

22 hrs

$V_{m}=-80 m V$

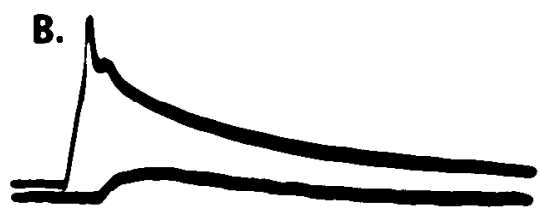

24 hrs

$$
\text { Ca-free Medium }
$$

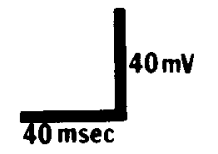

Figure 2. EPPs evoked in $(\Lambda)$ a myocyte from a standard culture and $(B)$ a myocyte from a Ca-free culture, by similar action potentials elicited in the presynaptic neurons. Injected current is not shown; current pulse was shorter for $B$ than for $A$. Recordings were made in standard saline. Upper trace, Action potential in neuron. Lower trace, EPP in myocyte. Note differences in sweep speed. In this and subsequent figures, $h r s$ refers to time in culture.

multiple, branched terminations, whereas contacts in Ca-free cultures lacked such endings (cf. Fig. 1, $A$ and $C$ ). In many cases, neurites would circumvent myocytes in their path (Fig. $1 B$ ) or would grow over them as a single unbranched neurite and end on cell-free areas of the dish (Figs. $1 C$ and $7 C$ ). To quantitate this difference, the number of neuron-myocyte contacts was determined for "target" myocytes in both standard and Ca-free media (a target myocyte being defined as one observed to be within $60 \mu \mathrm{m}$ of a neurite; Table I). Myocytes grown in Ca-free medium were contacted by neighboring neurites less frequently ( $57 \%$ for cells in Ca-free medium, $82 \%$ for cells in standard medium), and of those contacted, $29 \%$ displayed terminations compared to $89 \%$ observed in the standard cultures.

The differences in the pattern of neurite growth and neuronmyocyte contact observed in the two growth conditions suggested that functional transmission between neurons and myocytes in Ca-free cultures might be affected. Therefore, the number of functional contacts and the efficacy of synaptic transmission were examined for the different growth conditions.

Physiological recordings. Functional neuron-myocyte contacts formed with a high frequency in cultures grown in standard medium. Endplate potentials (EPPs) with variable amplitudes $(37.9 \pm 24.0 \mathrm{mV}$, mean $\pm \mathrm{SD})$ were evoked from $83 \%(15$ of 18) of all nerve-muscle pairs in which both cells were impaled with a microelectrode (Fig. $2 A$ ). Furthermore, small spontaneous EPPs were observed in 93\% (25 of 27) of neuritecontacted myocytes when only the myocyte was impaled (Fig. $3 A$ ). The times to peak for both spontaneous and evoked EPPs were similar (Table II). Both classes of EPPs could be reversibly blocked by bath application of $50 \mu \mathrm{M} d$-tubocurarine.
Functional neuron-myocyte connections were also observed in cultures grown in Ca-free medium (Fig. 2B); however, both the frequency and efficacy of synaptic contacts were significantly reduced compared to contacts between cells grown in standard medium. Only $28 \%$ (9 of 32 ) of cases in which both the neuron and contacted myocyte were impaled exhibited evoked EPPs. It seemed possible that impalement of the presynaptic cell could cause subtle retraction of synaptic contacts not apparent to visual inspection. However, only $25 \%$ ( 7 of 28 ) of the neuron-myocyte pairs in which the myocyte alone was impaled demonstrated spontaneous EPPs. This value is similar to that found for functional contacts assessed by double impalements (Fig. 3B). Neurites that did not display obvious morphological terminations nevertheless made functional contacts in these Ca-free cultures. Conversely, neurites that did display such terminations often did not.

Evoked potentials were smaller and slower than potentials recorded for myocytes grown in standard medium (Table II). The frequency of spontaneous events was significantly lower for myocytes grown in Ca-free medium $(0.24 \pm 0.5 / \mathrm{sec})$ than for those differentiating in standard medium $(2.04 \pm 1.6 / \mathrm{sec})$ (Table II, Fig. 4). As with cultures grown in standard medium, both spontaneous and evoked EPPs were reversibly blocked by $50 \mu \mathrm{M} d$-tubocurarine. Synaptic contacts formed on myocytes in $\mathrm{Ca}$-free cultures resembled conventional chemical synapses in that evoked EPPs were reversibly blocked by perfusion with a recording saline containing $0 \mathrm{~mm} \mathrm{CaCl}_{2} / 10 \mathrm{~mm} \mathrm{MgCl}_{2}$ (Fig. $5)$, although magnesium saline did not in all cases completely block spontaneous release. Spontaneous activity was present in $14 \%$ ( 2 of 14) of contacted myocytes grown in Ca-free medium and examined in magnesium saline. The frequency of the events, however, was extremely low: 0.01 and $0.06 / \mathrm{sec}$ in the two cells. Thus the mean frequency of spontaneous events from these 14 myocytes was at least 100 -fold less than that from myocytes grown in standard medium and examined in standard saline.

Differences in input resistance $\left(R_{\text {in }}\right)$ and resting potentials (RP) could potentially account for the differences in amplitude and time course of EPPs observed in the different growth conditions. Moreover, low $R_{\text {in }}$ and RP might mask detection of transmission that was present. No difference was seen in the mean RP for myocytes from the two culture conditions when recordings were made in standard saline. Myocytes in $\mathrm{Ca}$-free cultures did exhibit a lower mean $R_{\text {in }}$, but not significantly lower than that for myocytes from standard cultures (255 \pm 145 megohms, Ca-free cultures; $397 \pm 166$ megohms, standard cultures; $p<0.1$, two-tailed Student's $t$ test; values are mean $\pm \mathrm{SD}, N=10$ for each condition). Myocytes with low $R_{\text {in }}$, from both growth conditions, nevertheless exhibited spontaneous activity. In contrast, many myocytes with high $R_{\mathrm{in}}$, from $\mathrm{Ca}$ free cultures, did not.

Reductions in the efficacy of synapses between neurons and myocytes grown in the absence of calcium probably are due to a decrease in presynaptic release of neurotransmitter, to changes in postsynaptic sensitivity to ACh, or both. The distribution of $\mathrm{AChR}$ and sensitivity to $\mathrm{ACh}$ of myocytes from the two growth conditions were therefore investigated.

$\alpha-B G T$ labeling studies. In agreement with previous reports (Anderson and Cohen, 1977; Anderson et al., 1977), myocytes in cultures grown in standard medium and incubated with $\mathrm{K}$ $\alpha$-BGT exhibited numerous small, high density patches of fluorescently labeled AChR. AChR patches were observed on both neurite-contacted and noncontacted myocytes (Fig. 6, $B$ and $D$, Table III). On contacted myocytes, receptor patches were often, but not exclusively, localized to the immediate region of neuron-myocyte contact (Fig. 6, $A$ and $B$ ). In contrast, no high density $\mathrm{AChR}$ patches were observed on contacted myocytes grown in Ca-free medium ( $N=250$; Table III). 


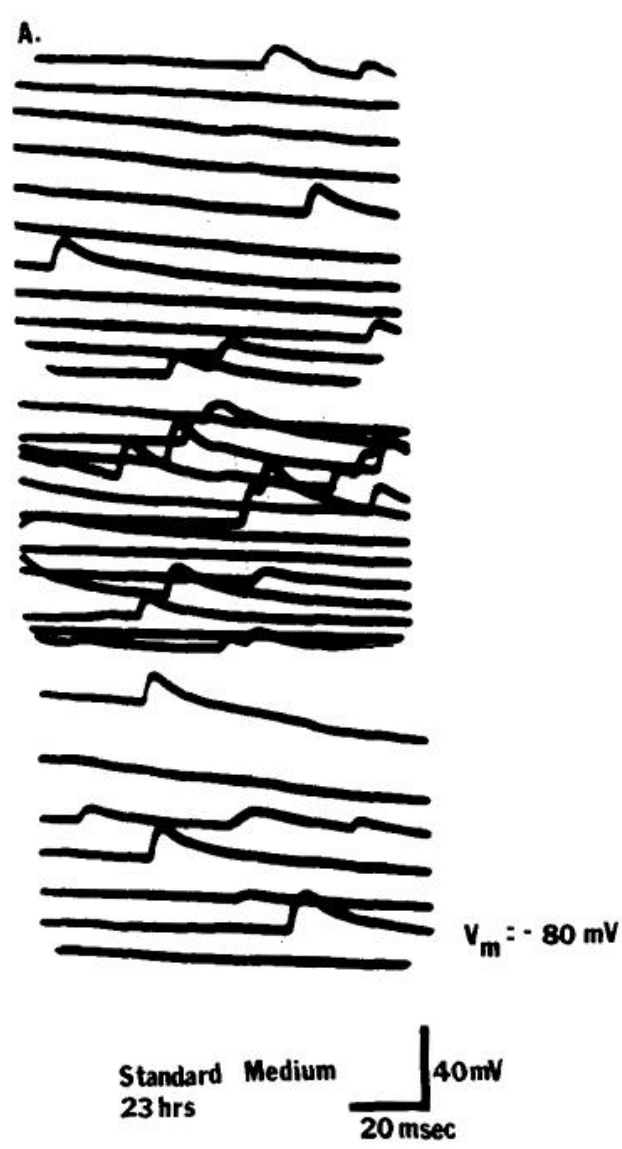

B.

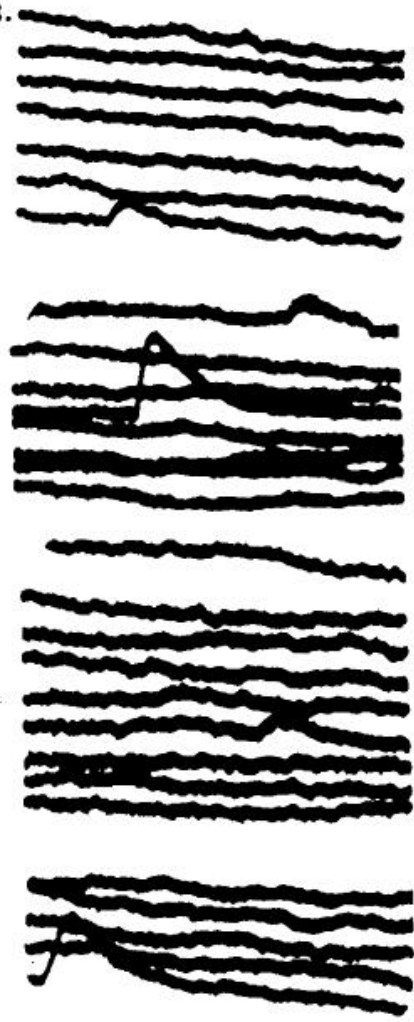

Ca-free Medium

25 hrs
Figure 3. Spontaneous potentials recorded from a myocyte in $(A)$ a standard culture and $(B)$ a Cafree culture. Sweeps are not consecutive for $B$. Note differences in gain and sweep speed. Recordings were made in standard saline.

TABLE II

Parameters of spontaneous and evoked potentials recorded from myocytes in standard and Ca-free cultures

Values are means \pm SD for the number of cells indicated. Frequency analysis was based on recordings made for 3 to 10 min. Recordings were made in standard saline. Values are significantly different, at the levels shown (one-tailed Student's $t$ test).

\begin{tabular}{|c|c|c|c|c|}
\hline \multirow[b]{2}{*}{ Medium } & \multirow{2}{*}{$\begin{array}{c}\text { Amplitude of } \\
\text { Evoked Potentials }\end{array}$} & \multicolumn{2}{|c|}{ Times to Peak } & \multirow{2}{*}{$\begin{array}{l}\text { Frequency of } \\
\text { Spontaneous } \\
\text { Potentials }\end{array}$} \\
\hline & & $\begin{array}{c}\text { Evoked } \\
\text { Potentials }\end{array}$ & $\begin{array}{l}\text { Spontaneous } \\
\text { Potentials }\end{array}$ & \\
\hline & $m V$ & \multicolumn{2}{|c|}{$m s e c$} & \\
\hline $\mathrm{Ca}^{2+}$-free medium & $\begin{array}{c}14.3 \pm 9.4 \\
(N=7) \\
p<0.025\end{array}$ & $\begin{array}{c}8.7 \pm 5.3 \\
(N=7) \\
p<0.1\end{array}$ & $\begin{array}{l}12.8 \pm 5.8 \\
(N=8) \\
p<0.001\end{array}$ & $\begin{array}{c}0.24 \pm 0.50 / \mathrm{sec} \\
(N=10) \\
p<0.001\end{array}$ \\
\hline
\end{tabular}

A.

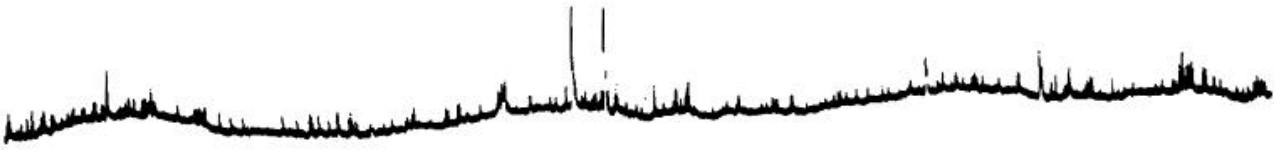

$V_{m}=-80 \mathrm{mV}$

Standard Medium

19 hrs

B.

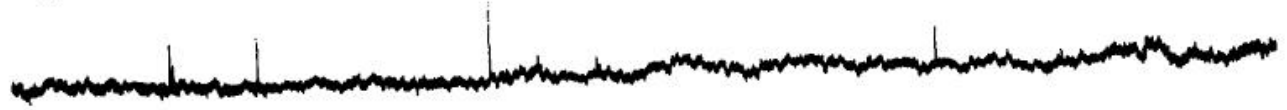

Figure 4. Spontaneous activity recorded from a myocyte in $(A)$ a standard culture and $(B)$ a $\mathrm{Ca}$ free culture. Only the myocytes, not the neurons, were impaled. Recordings were made in standard saline. $B$ represents the same cell as in Figure $3 B$.

Ca-free Medium 2 sec
25 hrs 
A.

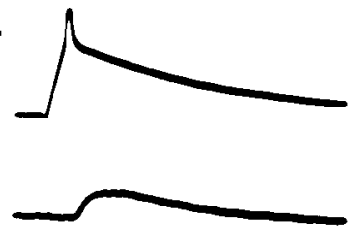

$10 \mathrm{mM} \mathrm{Ca} / 0 \mathrm{mM} \mathrm{Mg}$

B.

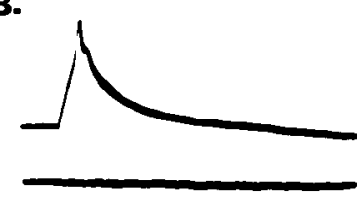

$0 \mathrm{mMCa} / 10 \mathrm{mMMg}$

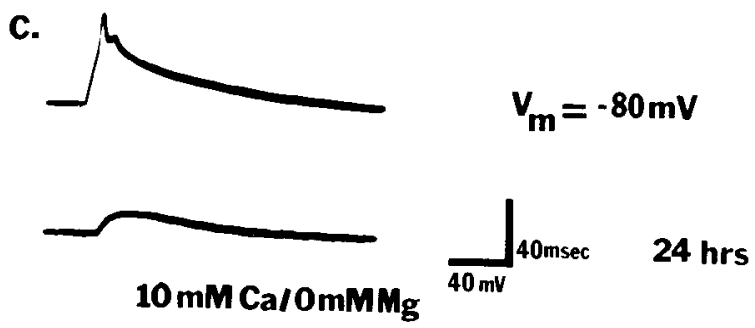

Figure 5. Saline in which magnesium replaces calcium reversibly blocks the EPP evoked in a myocyte grown in a Ca-free culture. Upper trace, Action potential in neuron. Lower trace, Response in myocyte. All records were from one pair of cells.

Experiments were made to examine the possibilities that high density AChR clusters might form by aggregation of existing receptors (Anderson and Cohen, 1977) or by insertion of new receptors (Frank and Fischbach, 1979) during the course of physiological experiments on myocytes grown in Ca-free medium. AChRs on myocytes in Ca-free medium were labeled with $\mathrm{R}-\alpha$-BGT; the Ca-free medium was then replaced with standard medium and cells were examined for fluorescent labeling at various times up to $24 \mathrm{hr}$. In cultures examined between 0 and $2 \mathrm{hr}$, only about $1 \%$ of myocytes exhibited high density AChR patches (Table III). It therefore seems unlikely that existing receptors in myocyte membranes undergo appreciable aggregation to form high density patches during a period corresponding to the longest time that cells were exposed to Ca-containing saline for intracellular recordings. Moreover, no $\mathrm{AChR}$ patches were observed on myocytes bathed in standard medium for $2 \mathrm{hr}$ before labeling with $\mathrm{R}-\alpha$-BGT $(N=200)$, suggesting that receptor patches consisting of newly inserted AChR do not appear during this period.

The distribution of silver grains in autoradiograms of cultures labeled with [ $\left.{ }^{125} \mathrm{I}\right] \mathrm{di}$-iodo- $\alpha$-RGT was consistent with the observations made using the fluorescently labeled toxin. In addition, radiolabeling of $\mathrm{AChR}$ indicates that, although myocytes grown in Ca-free medium lack high density AChR clusters, they nevertheless possess a low density, relatively uniformly distributed AChR population (Fig. 7). This population is similar in density to that of the evenly distributed AChR on myocytes in standard cultures (Fig. 8).

ACh sensilivily. All myocytes tested in standard and Ca-free cultures were sensitive to ACh (either bath applied or iontophoresed; $N=65$ ). When myocytes were tested with various concentrations of $\mathrm{ACh}$, the majority (31 of 33 ) were sensitive to $5 \mu \mathrm{M}$, and a few responded to $0.5 \mu \mathrm{M}$ (4 of 33 ). No differences were found in the sensitivity to bath-applied ACh of myocytes from standard and Ca-free cultures.
Regional differences in ACh sensitivity were assessed iontophoretically. In agreement with $\alpha$-BGT labeling studies, myocytes grown in standard medium exhibited discrete regions of high sensitivity to iontophoretically applied ACh (Fig. 9B). Regions of higher ACh sensitivity were more frequent at neurite-contacted than at noncontacted regions of myocyte membrane. The ratio of sensitivity of noncontacted to contacted myocyte membrane in standard cultures was $0.5 \pm 0.3(N=7$ myocytes; 3 to 10 sites per myocyte were sampled). Since AChR patches are not found at all regions of myocyte membrane contacted by neurites, this ratio is likely to be higher than that for extrajunctional versus junctional sensitivity. By comparison, myocytes differentiating in the absence of calcium were uniform in their sensitivity to $\mathrm{ACh}$ (Fig. 9A). The ratio of sensitivity of noncontacted to contacted regions was $1.2 \pm 0.3$ ( $N=8$ myocytes; 3 to 10 sites per cell were sampled).

Neurotransmitter sensitivity of neurons in Ca-free cultures. It is possible to identify neurons in culture grown in standard medium as resembling Rohon-Beard neurons or motor neurons on the basis of their sensitivity to various neurotransmitters (Nicoll et al., 1976; Bixby and Spitzer, 1982, 1984a). Motor neurons in vivo and in culture are depolarized by glutamate and hyperpolarized by GABA and glycine. The onset of neurotransmitter sensitivity is not affected by growing cells in the absence of calcium, although its later maturation is retarded (Bixby and Spitzer, 1984b). These results are extended by our observations that large numbers of neurons in these Ca-free cultures did not exhibit the normal profile of sensitivity. Many cells did not respond to either glutamate or GABA (glycine was not tested), whereas a small number responded to one transmitter but not to the other (Table IV). Neurons exhibiting unconventional sensitivity were nevertheless capable of making functional contacts with myocytes: two of five neurons tested that made functional contacts responded to glutamate, but not to GABA. The other three neurons responded to both neurotransmitters. No neurons contacting myocytes were found to exhibit the neurotransmitter sensitivity of Rohon-Beard cells.

\section{Discussion}

The simple growth requirements of differentiating Xenopus spinal cord neurons and myocytes have permitted us to investigate some of the requirements for synaptogenesis in culture and to assess the role of the calcium-dependent action potential in this process. We find that the establishment of functional neuromuscular contacts is not blocked by differentiation in the absence of calcium, although there is a substantial reduction in the frequency with which they are formed. Thus, neither the electrical activity nor the calcium influx associated with $\mathrm{Ca}$ dependent neuronal action potentials is required. Motor neurons differentiating in the absence of voltage-dependent calcium influx synthesize choline acetyltransferase and $\mathrm{ACh}$, package ACh in vesicles, and develop the synaptic machinery necessary for calcium-dependent transmitter release. Furthermore, the impulse-evoked release of $\mathrm{ACh}$ and other vesicular contents is not necessary for the initial formation of functional connections, although trophic factors may be involved in subsequent development of synapses. A role for spontaneous or nonvesicular release in synaptogenesis is not excluded by our findings. These results are complementary to those of Camardo et al. (1983), who reported that the release of neurotransmitter and the presence of appropriate receptors are not sufficient conditions for the formation of functional synaptic contacts between identified Aplysia neurons in culture.

We cannot rule out the possibility that small potentials were lost in the base line noise in some of the cases in which transmission was judged to be absent. However, when low resistance electrodes were used, the noise was often less than $500 \mu \mathrm{V}$ peak to peak when recording from cells whose input 

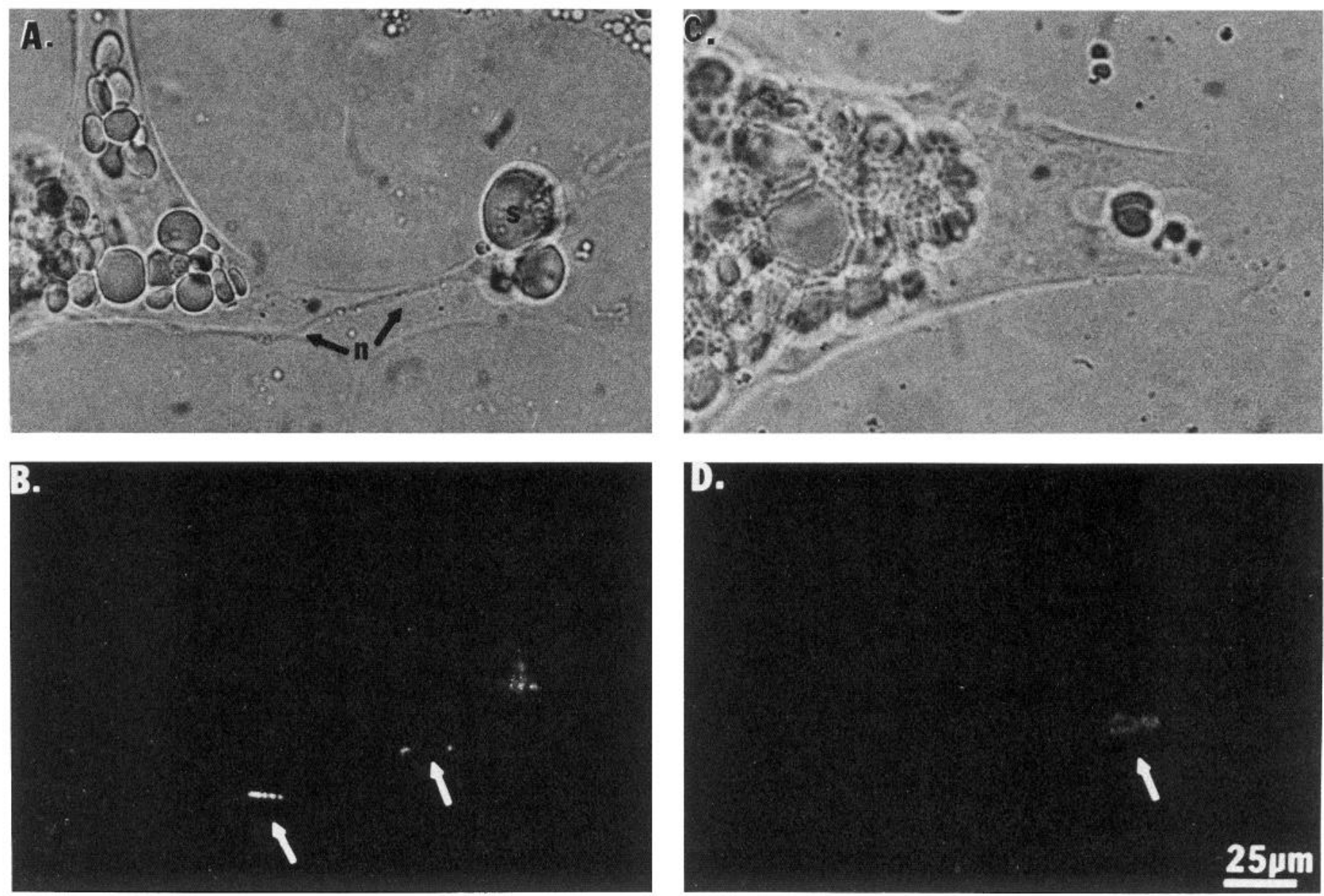

Figure 6. R- $\alpha$-BGT-labeled AChR patches on myocytes grown in standard medium. Transmitted light $(A)$ and fluorescence micrographs $(B)$ of the same field showing R- $\alpha$-BGT-labeled AChR patches at sites of neurite contact on a myocyte. Transmitted light $(C)$ and fluorescence micrographs $(D)$ of labeled AChR patches on a noncontacted myocyte. All micrographs are at $26 \mathrm{hr}$ in culture. $n$, neurite; $s$, soma. White arrows indicate fluorescent $\mathrm{AChR}$ patches.

\section{TABLE III}

$R-\alpha-B G T$ binding to cultured myocytes

Cultures were plated in either standard or Ca-free medium and later were incubated for $1 \mathrm{hr}$ in R- $\alpha$-BGT. Some Ca-free cultures were subsequently exposed to standard medium. Cells were examined using fluorescence optics.

\begin{tabular}{|c|c|c|c|}
\hline Cultures & No. of Myocytes with Hot Spots & $\begin{array}{c}\text { No. of Hots Spots } \\
\text { per Myocyte } \\
\text { (mean } \pm \text { SD) }\end{array}$ & $\begin{array}{l}\text { Time Exposed to } \\
\text { Standard } \\
\text { Medium } \\
\end{array}$ \\
\hline $\begin{array}{l}\text { Standard cultures } \\
\text { Ca-free cultures }\end{array}$ & $\begin{array}{c}\text { Contacted and Noncontacted } \\
44(N=50) \\
\text { Contacted } \\
0(N=250) \\
\text { Contacted and Noncontacted } \\
0(N=52) \\
2(N=75) \\
0(N=50) \\
10(N=100)\end{array}$ & $3.8 \pm 3.1$ & $\begin{array}{c}0 \mathrm{~min} \\
15 \mathrm{~min} \\
1 \mathrm{hr} \\
2 \mathrm{hr} \\
24 \mathrm{hr}^{a}\end{array}$ \\
\hline
\end{tabular}

resistances were approximately 200 megohms. Given the large amplitudes of potentials in such cells when transmission was present, it seems likely that functional synaptic contacts were truly absent when no responses were observed.

Several observations suggest that neuron-myocyte contacts formed in the absence of calcium achieve only a limited level of differentiation. Spontaneous responses recorded at 1 day in culture were of low frequency, as has been reported for initial neuromuscular contacts in Xenopus nerve-muscle cultures (Anderson et al., 1979; Kidokoro et al., 1980). In addition, we also found that, in comparison to cells grown in standard medium, a reduction in mean amplitude was evident in recordings from neuron-myocyte pairs grown in $\mathrm{Ca}$-free medium.

It seems unlikely that cells grown in this medium are simply retarded in their general development. Myocytes differentiating in Ca-free medium develop cross-striations and are able to generate action potentials and contract when bathed with normal saline. Autoradiography and $\mathrm{ACh}$ sensitivity measurements indicate that the levels of $\mathrm{AChR}$ synthesized and inserted into myocyte membrane are comparable to the levels of evenly distributed AChR on myocytes from standard cultures; a small decrease could have gone undetected (McManaman et al., 

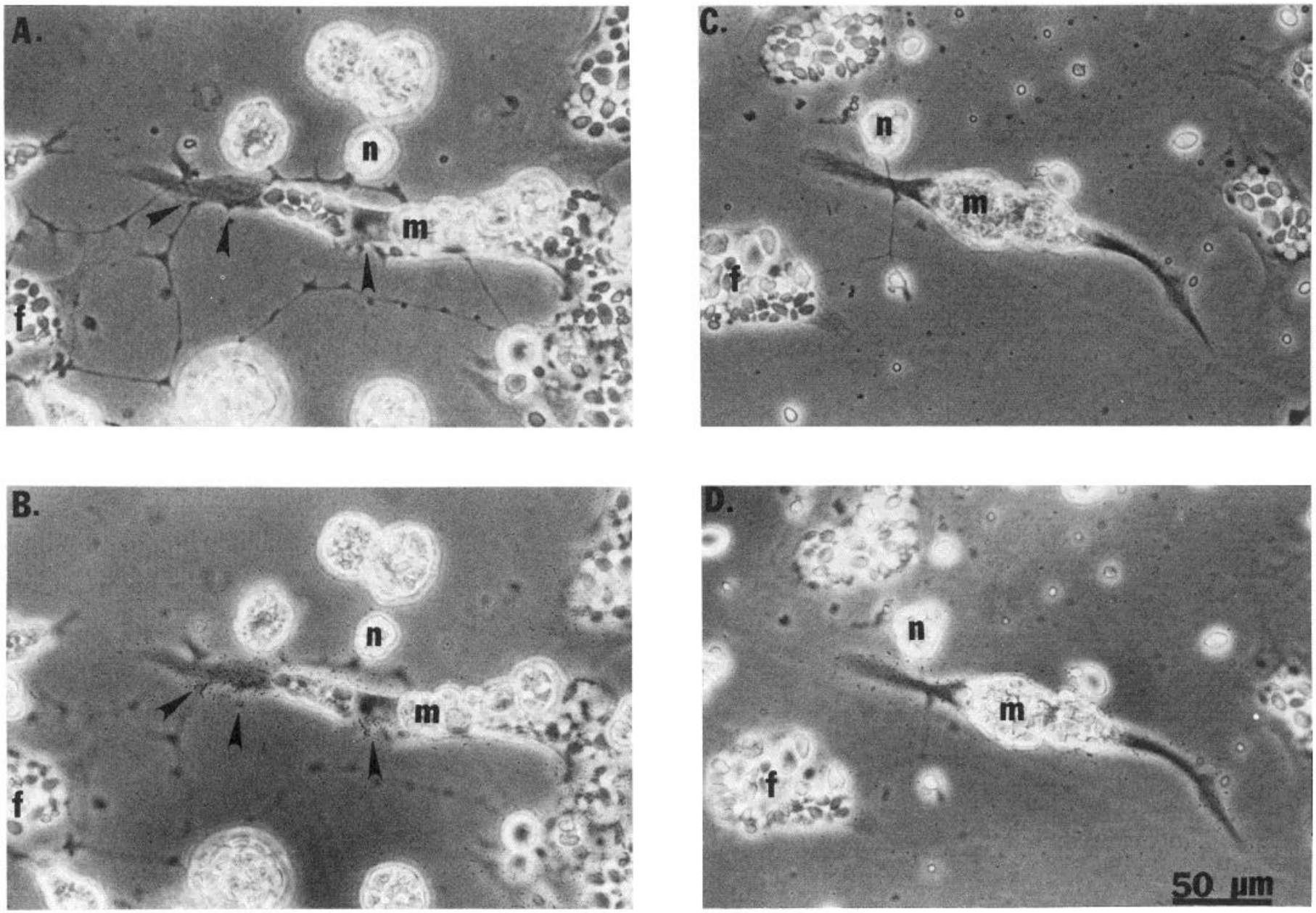

Figure 7. AChR distribution on myocytes from different growth conditions. $A$ and $B$, Phase micrographs of an autoradiogram showing a neurite-contacted myocyte $(\mathrm{m})$ grown in standard medium and labeled with [ $\left.{ }^{125} \mathrm{I}\right]$ di-iodo- $\alpha$-BGT. $B$ shows the same field as in $A$ with the plane of focus adjusted to reveal the distribution of silver grains. Patches of high AChR density (arrowheads) are associated with neurite-contacted regions of the myocyte. $n$, neuronal soma. $C$ and $D$, Phase contrast micrographs and silver grain distribution in an autoradiogram of a neuritecontacted myocyte grown in Ca-free medium. AChRs are present at a uniform density over the surface of the myocyte. Levels of nonspecific binding, observed when labeling was performed in the presence of a 100 -fold excess of unlabeled $\alpha$-BGT, were similar to the grain density in areas of the culture lacking myocytes. Note that fibroblast-like cells $(f)$ in both culture conditions do not show grain densities above background. Micrographs were taken at $24 \mathrm{hr}$ in culture.
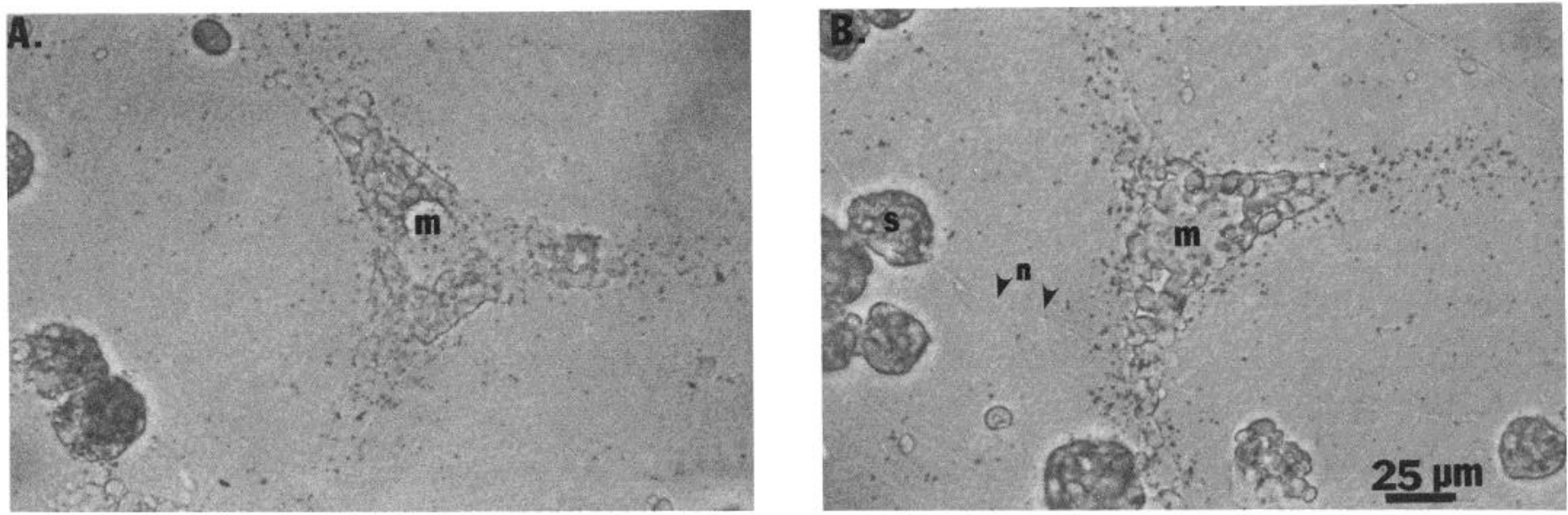

Figure 8. AChR densities on myocytes from standard and Ca-free cultures. Brightfield micrographs of $(A)$ a noncontacted myocyte in standard medium and $(B)$ a contacted myocyte in Ca-free medium labeled with $\left[{ }^{125} \mathrm{I}\right]$ di-iodo- $\alpha$-BGT. Densities of the grains from the population of evenly distributed AChRs are similar. Arrowheads in $B$ indicate neurite $(n) . s$, neuron soma; $m$, myocyte. Micrographs were taken at $26 \mathrm{hr}$ in culture. 

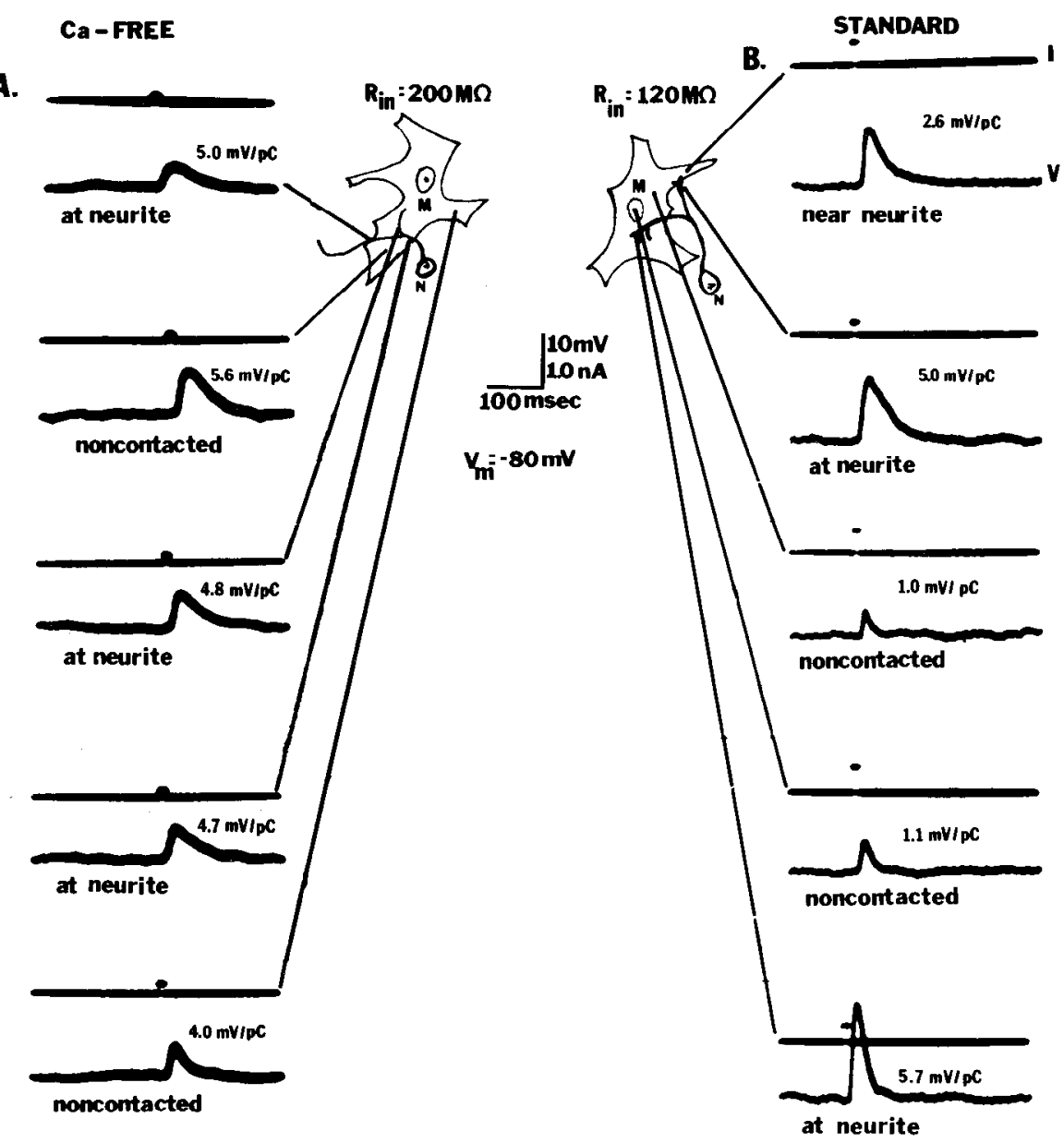

Figure 9. Regional sensitivity of two myocytes to iontophoretically applied ACh. A Cafree culture $(A)$ and a standard culture $(B)$. Recordings were made in standard saline. The sensitivity for $A$ was calculated according to the procedure described under "Materials and Methods." The sensitivity for $B$ was also calculated by this procedure, but to illustrate the regional differences in sensitivity of myocytes in standard cultures, responses larger than 5 $\mathrm{mV}$ are shown here. Sensitivities were not corrected for differences in $R_{\text {in }}$. Regions designated at neurite or near neurite were within $5 \mu \mathrm{m}$ of the neurite. Cells were approximately $24 \mathrm{hr}$ in culture. $I$, iontophoretic current; $V$, membrane potential. $R_{\text {pipetre }}=1600$ megohms $(A)$ and 1400 megohms $(B) ; I_{\text {brake }}=-0.15 \mathrm{nA}(A)$ and -0.3 $\mathrm{nA}(B)$.

TABLE IV

The neurotransmitter sensitivity of neurons contacting myocytes at $\sim 1$ day in Ca-free cultures

Sensitivity was assayed by bath application of $100 \mu \mathrm{M}$ glutamate and GABA. All neurons in standard cultures respond to GABA and $70 \%$ of neurons respond to glutamate by this age (Bixby and Spitzer, 1984a).

\begin{tabular}{cccc}
\hline +Glutamate/+GABA & -Glutamate/-GABA & -Glutamate/+GABA & -Glutamate/-GABA \\
\hline $31 / 70$ & $17 / 70$ & $3 / 70$ & $19 / 70$ \\
$44 \%$ & $24 \%$ & $4 \%$ & $28 \%$ \\
\hline
\end{tabular}

${ }^{a}+$, neuron responds to transmitter. - , neuron does not respond to transmitter.

1981). Spinal cord neurons differentiating in Ca-free medium extend neurites and develop calcium- and sodium-dependent action potentials at the same stage they would have in standard cultures and in vivo (Bixby and Spitzer, 1984b). The initial formation of neuron-myocyte contacts, in contrast to subsequent differentiation, is similar to these other early developmental events in that the presence of extracellular calcium is not required.

The reduction in efficacy of functional synaptic contacts can be attributed partly to postsynaptic effects. In agreement with previous reports (Anderson and Cohen, 1977; Anderson et al., 1977; Kidokoro and Gruener, 1982), high density patches of AChR were observed at neurite-contacted and noncontacted regions of myocytes grown in standard medium. In contrast, no $\mathrm{AChR}$ patches were observed on myocytes grown in Ca-free medium, even at sites contacted by neurites. The establishment of functional neuromuscular contacts in the absence of high density receptor clusters, with properties similar to those reported here, has been observed both in vitro (Anderson el al., 1979; Kidokoro et al., 1980) and in vivo (Blackshaw and Warner, 1976; Bevan and Steinbach, 1977; Kullberg et al., 1977; Dennis et al., 1981; Chow and Cohen, 1983). We do not know the cause of the reduction in the number of neuron-myocyte contacts and neurite terminations on myocytes. Calcium appears to play an important role in the ability of neurons to recognize myocytes as appropriate targets, since neurites in calcium-free cultures often avoided myocytes directly in their path. This phenomenon is not simply attributable to the lack of AChR clusters on myocytes in calcium free medium, since it has been shown that pre-existing receptor patches do not represent preferred sites for initial neuromuscular contacts (Anderson and Cohen, 1977).

Information about the mechanisms underlying the generation of $\mathrm{AChR}$ clusters is provided by their failure to appear at neurite-contacted sites on myocytes grown in Ca-free medium, despite the establishment of functional connections. Cangiano et al. (1980) have shown that motor neurons in which impulse activity is blocked readily make new synapses on previously innervated muscle and that transmission at these synapses is similar to that observed at synapses made by active neurons. Furthermore, chronic blockade of receptor function does not prevent $\mathrm{AChR}$ aggregation at nerve-muscle contacts during synaptogenesis (Anderson and Cohen, 1977; Anderson et al., 1977). It seems likely, therefore, that AChR clustering at both 
nerve-contacted and noncontacted sites during initial synaptogenesis, as well as in reinnervation, is a $\mathrm{Ca}$-dependent process and is not dependent on presynaptic action potentials or postsynaptic ACh-induced clectrical activity. Recent studies indicate that $\mathrm{AChR}$ clusters on rat myotubes in tissue culture (Bloch, 1983; Bursztajn et al., 1984) and on embryonic rat sternomastoid muscles fibers in organ culture (Bloch and Steinbach, 1981) are reversibly lost upon depletion of calcium from the culture medium, and that Ca-free medium suppresses the formation of AChR clusters (Peng, 1984). The experiments presented here indicate that the presence of a physical neuromuscular contact is not sufficient to override this suppression.

Concomitant with normal development of postsynaptic specializations, there is a change in presynaptic function: the frequency of spontaneous potentials increases (Diamond and Miledi, 1962; Kidokoro, 1980; Kidokoro et al., 1980; Nakajima et al., 1980; Dennis et al., 1981). It appears that the lack of calcium prevents some aspects of presynaptic as well as postsynaptic maturation, since the frequency of spontaneous potentials recorded from myocytes in Ca-free cultures in standard saline is roughly one-tenth that recorded from myocytes in standard cultures. Ceccarelli et al. (1979) have shown that acute treatment of adult frog nerve-muscle preparations with calcium-free medium disrupts the organization of active zones, although black widow spider venom-evoked release of $\mathrm{ACh}$ is unaffected.

Lack of normal neuronal neurotransmitter sensitivity is not associated with the absence of synaptic transmission. Standard cultures prepared from stage 15 embryos contain primarily Rohon-Beard cells and motor neurons (Spitzer and Lamborghini, 1976; Lamborghini, 1980). These neurons respond to GABA and to GABA and glutamate, respectively, and no neurons are insensitive to GABA at 1 day in culture (Bixby and Spitzer, 1982, 1984a). Many neurons in Ca-free cultures were insensitive to GABA or to both transmitters. It is unlikely that these results can be attributed to differential survival of subpopulations of neurons, as .J. F. Tamborghini (personal communication) has found that the Ca-free cultures contain the same percentage of early birth date neurons as that found in $\mathrm{Ca}$ containing cultures. Neurons which were not sensitive to GABA, but did respond to glutamate, formed functional synaptic contacts on myocytes. In addition, neurons insensitive to both transmitters formed functional chemical contacts with other neurons (unpublished observation). It seems likely that neurons in Ca-free cultures retain the ability to synthesize their proper transmitter (in the case of motor neurons, ACh), but that their sensitivity to neurotransmitters is different from that of neurons of the same age in standard cultures. In contrast, all myocytes tested from Ca-free cultures were sensitive to $\mathrm{ACh}$.

It is probable that the lack of physiological "maturity" of synapses in these Ca-free cultures reflects an unspecialized ultrastructure. At 1 day in culture, the ultrastructure of regions of contact in standard cultures is variable. Clefts are often nonuniform and basal lamina is sparse. Vesicle clusters may or may not be present, and active zones are rare (Weldon and Cohen, 1979), yet some specializations are evident (Peng et al., $1979,1980)$. It would be of interest to examine the ultrastructure of junctions of myocytes in Ca-free cultures both at 1 day and at later times in culture. Such experiments will necessitate changing the culture medium used in these experiments since no glucose or sera were included, and cells in Ca-free cultures often exhausted their endogenous energy supply and disappeared by the second day in culture.

\section{References}

Anderson, M. J., and M. W. Cohen (1977) Nerve-induced and spontaneous redistribution of acetylcholine receptors on cultured muscle cells. J. Physiol. (Lond.) 268: 757-773.
Anderson, M. J., M. W. Cohen, and E. Zorytcha (1977) Effects of innervation on the distribution of acetylcholine receptors on cultured muscle cells. J. Physiol. (Lond.) 268: 731-756.

Anderson, M. J., Y. Kidokoro, and R. Gruener (1979) Correlation between acetylcholine receptor localization and spontaneous synaptic potentials in cultures of nerve and muscle. Brain Res. 166: 185190.

Baccaglini, P. I., and N. C. Spitzer (1977) Developmental changes in the inward current of the action potential of Rohon-Beard neurones. J. Physiol. (Lond.) 271: 93-117.

Bennett, M. R., and A. G. Pettigrew (1974) The formation of synapses in striated muscle during development. J. Physiol. (Lond.) 241: 515545.

Bevan, S., and J. H. Steinbach (1977) The distribution of $\alpha$-bungarotoxin binding sites on mammalian skeletal muscle developing in vivo. J. Physiol. (Lond.) 267: 195-213.

Bixby, J. L., and N. C. Spitzer (1982) The appearance and development of chemosensitivity in Rohon-Beard neurones of the Xenopus spinal cord. J. Physiol. (Lond.) 330: 513-536.

Bixby, J. L., and N. C. Spitzer (1984a) The appearance and development of neurotransmitter sensitivity in embryonic Xenopus spinal neurons in vitro. J. Physiol. (Lond.) 353: 143-155.

Bixby, J. L., and N. C. Spitzer (1984b) Early differentiation of vertebrate spinal neurons in the absence of voltage-dependent $\mathrm{Ca}^{++}$and $\mathrm{Na}^{+}$influx. Dev. Biol. 106: 89-96.

Blackshaw, S. E., and A. E. Warner (1976) Onset of acetylcholine sensitivity and endplate activity in developing myotome muscles of Xenopus. Nature 262: 217-218.

Blair, L. A. C. (1983) The timing of protein synthesis required for the development of the sodium action potential in embryonic spinal neurons. J. Neurosci. 3: 1430-1436.

Bloch, R. .J. (1983) Acetylcholine receptor clustering in rat myotubes: Requirement for $\mathrm{Ca}^{2+}$ and effects of drugs which depolymerize microtubules. J. Neurosci. 3: 2670-2680.

Bloch, R. J., and J. H. Stcinbach (1981) Reversible loss of acetylcholine receptor clusters at the developing rat neuromuscular junction. Dev. Biol. 81: 386-391.

Buckley, K. M., E. S. Schweitzer, G. P. Miljanich, L. Clift-O'Grady, P. D. Kushner, L. F. Reichardt, and R. B. Kelly (1983) A synaptic vesicle antigen is restricted to the junctional region of the presynaptic plasma membrane. Proc. Natl. Acad. Sci. U. S. A. 80: 7342-7346.

Bursztajn, S., J. L. McManaman, and S. H. Appel (1984) Organization of acetylcholine receptor clusters in cultured rat myotubes is calcium dependent. J. Cell Biol. 98: 507-517.

Camardo, J., E. Proshansky, and S. Schacher (1983) Identified Aplysia neurons form specific chemical synapses in culture. J. Neurosci. 3: $2614-2620$.

Cangiano, A., T. Lømo, L. Lutzemberger, and O. Sveen (1980) Effects of chronic nerve conduction block on formation of neuromuscular junctions and junctional acetylcholinesterase in the rat. Acta Physiol. Scand. 109: 283-296.

Ceccarelli, B., F. Grohovaz, and W. P. Hurlbut (1979) Freeze-fracture studies of frog neuromuscular junctions during intense release of neurotransmitter. J. Cell Biol. 81: 163-177.

Chow, I., and M. W. Cohen (1983) Developmental changes of acetylcholine receptors in the myotomes of Xenopus laevis. J. Physiol. (Lond.) 339: 553-571.

Cohen, M. W. (1972) The development of neuromuscular connexions in the presence of D-tubocurarine. Brain Res. 41: 457-463.

del Castillo, J., and B. Katz (1954) Quantal components of the endplate potential. J. Physiol. (Lond.) 124: 560-573.

Dennis, M. J., L. Ziskind-Conhaim, and A. J. Harris (1981) Development of neuromuscular junctions in rat embryos. Dev. Biol. 81: 266279.

Diamond, J., and R. Miledi (1962) A study of foetal and new-born rat muscle fibres. J. Physiol. (Lond.) 162: 393-408.

Douglas, W. W., and A. M. Poisner (1966) On the relationship between ATP splitting and secretion in the adrenal chromaffin cells: Discharge of ATP (unhydrolyzed) during release of catecholamines. J. Physiol. (Lond.) 179: 130-137.

Frank, E., and G. D. Fischbach (1979) Early events in neuromuscular junction formation in vitro. Induction of acetylcholine receptor clusters in the postsynaptic membrane and morphology of newly formed synapses. J. Cell Biol. 83: 143-158.

Fukuda, J., and M. Kameyama (1979) Enhancement of Ca spikes in 
nerve cells of adult mammals during neurite growth in tissue culture. Nature 279: 546-548.

Goodman, C. S., and N. C. Spitzer (1979) Embryonic development of identified neurons: Differentiation from neuroblast to neurone. $\mathrm{Na}$ ture 280: 208-214.

Gruener, R., and Y. Kidokoro (1982) Acetylcholine sensitivity of innervated and noninnervated Xenopus muscle cells in culture. Dev. Biol. 91: 86-92.

Hume, R. I., L. W. Role, and G. D. Fischbach (1983) Acetylcholine release from growth cones detected with patches of acetylcholinereceptor rich membranes. Nature 305: 632-634.

Jansen, J. K. S., and D. C. Van Essen (1975) Re-innervation of rat skeletal muscle in the presence of $\alpha$-bungarotoxin. J. Physiol. (Lond.) 250: 651-667.

Kidokoro, Y. (1980) Developmental changes of spontaneous synaptic potential properties in the rat neuromuscular contact formed in culture. Dev. Biol. 78: 231-241.

Kidokoro, Y., and R. Gruener (1982) Distribution and density of $\alpha$ bungarotoxin binding sites on innervated and noninnervated Xenopus muscle cells in culture. Dev. Biol. 91: 78-85.

Kidokoro, Y., and E. Yeh (1982) Initial synaptic transmission at growth cones in Xenopus nerve-muscle cultures. Proc. Natl. Acad. Sci. U. S. A. 79: $6727-6731$.

Kidokoro, Y., M. J. Anderson, and R. Gruener (1980) Changes in synaptic potential properties during acetylcholine receptor accumulation and neurospecific interactions in Xenopus nerve-muscle cell culture. Dev. Biol. 78: 464-483.

Kullberg, R. W., T. L. Lentz, and M. W. Cohen (1977) Development of the myotomal neuromuscular junction in Xenopus laevis: An electrophysiological and fine-structural study. Dev. Biol. 60: 101129.

Lamborghini, J. E. (1980) Rohon-Beard cells and other large neurons in Xenopus embryos originate during gastrulation. J. Comp. Neurol. 189: 323 -333.

Matsuda, Y., S. Yoshida, and T. Yonezawa (1976) A Ca-dependent regenerative response in rodent dorsal root ganglion cells cultured in vitro. Brain Res. 115: 334-338.

McManaman, J. L., J. C. Rlosser, and S. H. Appel (1981) The effect of calcium on acetylcholine receptor synthesis. J. Neurosci. 1: 771-776.

Meiri, H., M. E. Spira, and I. Parnas (1981) Membrane conductance and action potential of a regenerating axonal tip. Science 211: 709712 .

Miyake, M. (1978) The development of action potential mechanism in a mouse neuronal cell line in vilro. Brain Res. 143: 349-354.

Mori-Okamoto, J., M. Ashida, E. Maru, and J. Tatsuno (1983) The development of action potentials in cultures of explanted cortical neurons from chick embryos. Dev. Biol. 97: 408-416.

Musick, J., and J. I. Hubbard (1972) Release of protein from mouse motor nerve terminals. Nature 237: 279-281.

Nakajima, Y., Y. Kidokoro, and F. G. Klier (1980) The development of functional neuromuscular junctions in vitro: An ultrastructural and physiological study. Dev. Biol. 77: 52-72.

Nicoll, R. A., A. Padjen, and J. L. Barker (1976) Analysis of amino acid responses on frog motoneurones. Neuropharmacology 15: 4553.

Nieuwkoop, P. D., and J. Faber (1956) Normal Table of Xenopus laevis (Daudin), Elsevier-North Holland Publishing Co., Amsterdam.

O'Dowd, D. K. (1983) RNA synthesis dependence of action potential development in spinal cord neurones. Nature 303: 619-621.

Peng, H. B. (1984) Participation of calcium and calmodulin in the formation of acetylcholine receptor clusters. J. Cell Biol. 98: 550557.

Peng, H. B., P. C. Bridgman, S. Nakajima, A. Greenberg, and Y. Nakajima (1979) A fast development of presynaptic function and structure of the neuromuscular junction in Xenopus tissue culture. Brain Res. 167: 379-384.

Peng, H. B., Y. Nakajima, and P. C. Bridgman (1980) Development of the postsynaptic membrane in Xenopus neuromuscular cultures observed by freeze-fracture and thin-section electron microscopy. Brain Res. 196: 11-31.

Ravdin, P., and D. Axelrod (1977) Fluorescent tetramethylrhodamine derivatives of $\alpha$-bungarotoxin: Preparation, separation and characterization. Anal. Biochem. 80:585-592.

Ravdin, P. M., and D. K. Berg (1979) Inhibition of neuronal acetylcholine sensitivity by $\alpha$-toxin from Bungarus multicinctus venom. Proc. Natl. Acad. Sci. U. S. A. 76: 2072-2076.

Rubin, L. L., S. M. Schuetze, C. L. Weill, and C. D. Fischbach (1980) Regulation of acetylcholinesterase appearance at neuromuscular junctions in vitro. Nature 283: 264-267.

Spitzer, N. C. (1983) The development of neuronal membrane properties in vivo and in culture. In Developing and Regenerating Vertebrate Nervous Systems, P. W. Coates, R. R. Markwald, and A. D. Kenny, eds., pp. 41-59, Alan R. Liss, lnc., New York.

Spitzer, N. C., and J. E. Lamborghini (1976) The development of the action potential mechanism of amphibian neurons isolated in culture. Proc. Natl. Acad. Sci. U. S. A. 73: 1641-1645.

Steinbach, J. H., A. J. Harris, J. Patrick, D. Schubert, and S. Heinemann (1973) Nerve-muscle interaction in vitro: Role of acetylcholine. J. Gen. Physiol. 62: 255-270.

Vogel, A., A. J. Sytkowski, and M. W. Nirenberg (1972) Acetylcholine receptors of muscles grown in vitro. Proc. Natl. Acad. Sci. U. S. A. 69: $3180-3184$.

Weldon, P. R., and M. W. Cohen (1979) Development of synaptic ultrastructure at neuromuscular contacts in an amphibian cell culture system. J. Neurocytol. 8: 239-259.

Willard, A. L. (1980) Electrical excitability of outgrowing neurites of embryonic neurons in cultures of dissociated neural plate of Xenopus laevis. J. Physiol. (Lond.) 301: 115-128.

Young, S. H., and M. Poo (1983) Spontaneous release of transmitter from growth cones of embryonic neurons. Nature 305: 634-637. 